Edited by Kiriakos Xenitidis and
Colin Campbell
Contents
- Confectionery consumption and violence
- Structural brain abnormalities in bipolar disorder
- Evolution and psychiatry

\section{Confectionery consumption and violence}

Moore et al ${ }^{1}$ found a 'novel and robust' relationship between confectionery consumption during childhood and conviction for violence in adulthood. However, there are serious methodological concerns, which make the overall findings questionable. As the authors recognise, the number of violent people in their cohort is very low. The lack of descriptive information in the paper, contrary to recommendations on the reporting of observational studies, ${ }^{2}$ forces the reader to an exercise of reconstruction. What emerges is that only about 33 participants were violent $(0.47 \%$ of 6942 ) and of these, only 23 (69\% of the 33 violent individuals) had eaten confectionery excessively. With such numbers, it is highly discouraged in the biostatistical literature to model the probability of being violent using as many parameters (8) as the authors did, since the fit is essentially driven by the number of cases and not by the entire sample size. ${ }^{3}$ The deficiencies of this approach are well known and numerous, affecting all aspects of the modelling process, from variable selection to effect size estimation, ${ }^{4}$ and are not, generally, accommodated by the adoption of rare-events logistical models, which only provide a fix for bias in estimating regression parameters. With such few cases, no interactions have been considered, even though some may be very intuitive (e.g. confectionery consumption and child-oriented parenting). With no serious attempt at considering interactions in the model, the risk of finding spurious associations is well documented (Simpson's paradox). ${ }^{5}$ Unfortunately, no details are provided in the paper concerning distribution of the other seven factors included in the multivariable model (gender, late birth, etc.) between violent and non-violent people, so that it is almost impossible to understand how low the cell frequency is in some such combinations. With these considerations in mind, the conclusions suggesting a relationship between confectionery and violence seem an over-interpretation of the fitted model.

1 Moore SC, Carter LM, van Goozen SHM. Confectionery consumption in childhood and adult violence. Br J Psychiatry 2009; 195: 366-7.

2 Vandenbroucke JP, von Elm E, Altman DG, Gotzsche PC, Mulrow CD, Pocock $\mathrm{SJ}$, et al. Strengthening the Reporting of Observational Studies in Epidemiology (STROBE): explanation and elaboration. Epidemiology 2007; 18: $805-35$.

3 Harrell FE Jr, Lee KL, Mark DB. Multivariable prognostic models: issues in developing models, evaluating assumptions and adequacy, and measuring and reducing errors. Stat Med 1996; 15: 361-87.

4 Harrell FE Jr, Lee KL, Califf RM, Pryor DB, Rosati RA. Regression modelling strategies for improved prognostic prediction. Stat Med 1984; 3: 143-52.

5 Agresti A. Categorical Data Analysis (2nd edn). Wiley \& Sons, 2002.

D. Gregori, Department of Environmental Medicine and Public Health, University of Padova, Italy. Email: dario.gregori@unipd.it

doi: 10.1192/bjp.196.3.245
Author's reply: We take issue with Gregori's statement that methodological concerns render the overall findings of our report ${ }^{1}$ questionable. Gregori correctly observes that 'the number of violent people in their cohort is very low' and goes on to suggest that reporting results on such small samples should be discouraged. We are interested in life-course factors that predict adult violence in the hope that such research might inform early life-course interventions. We therefore have two options. Either recruit violent offenders and enquire of their childhoods, or follow a cohort of individuals recording information on their circumstances to assess associations with later problem behaviour. Unfortunately, compounding the vagaries of human memory are the particular difficulties many offenders have with recalling what they did the previous day, let alone several decades ago. It is thus unfeasible to conduct retrospective studies; this leaves cohort studies as the only realistic and robust methodology. We are fortunate in the UK to have one of the most highly regarded cohort studies in the world, but despite its large initial sample size the rarity of violence means that only a small number of respondents demonstrate the behaviour of interest. Should we, as Gregori counsels, simply not consider using the British Cohort Study to look into childhood factors predicting adult violence because violence is rare? We suggest that this would be a valuable and informative resource squandered if that advice were followed. Gregori also suggests that models on rare data should not involve too many covariates. In our short paper we reported that we considered various configurations including the unadjusted association between confectionery and violence and that the strength of association was consistent across models - analytically we did as much as we could to test this association. We chose not to report simpler models and hardly mentioned the extensive analyses assessing the impact of attrition simply because we felt this paper suited a short-report format and including this additional information would only detract from what was a perfectly well-articulated finding. We therefore maintain that we analysed some of the best cohort data available to assess childhood predictors of an important outcome and found a robust association. We were honest with regard to the sample size, concluding in the paper that this is one area that should be addressed before firm conclusions can be drawn.

1 Moore SC, Carter L, Van Goozen SHM. Confectionery consumption in childhood and adult violence. Br J Psychiatry 2009; 195: 366-7.

Simon C. Moore, Violence and Society Research Group, Applied Clinical Research and Public Health, School of Dentistry, University of Cardiff, Cardiff CF14 4XY, UK. Email: mooresc2@cardiff.ac.uk

doi: $10.1192 / \mathrm{bjp} .196 .3 .245 a$

\section{Structural brain abnormalities in bipolar disorder: what meta-analyses tell us}

Findings from Arnone et al's ${ }^{1}$ systematic review and meta-analysis of magnetic resonance imaging (MRI) studies suggest that patients with bipolar disorder are characterised, in comparison with healthy controls, by significant reductions of whole-brain and prefrontal lobe volumes and by enlargement of lateral ventricles and globus pallidus, although most of the brain changes detected in bipolar disorder do not seem to be diagnostically specific and some clinical variables, such as patients' age, duration of illness and pharmacological treatment, appear to be relevant in determining the magnitude of observed effect sizes.

These findings are not completely consistent our own recent meta-analysis ${ }^{2}$ of MRI studies in first-episode bipolar disorder. 